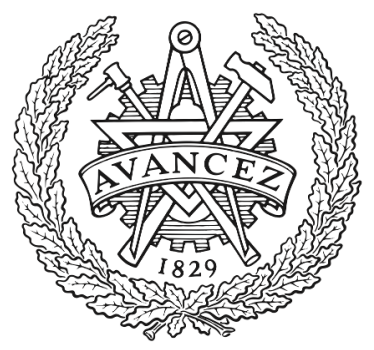

CHALMERS

UNIVERSITY OF TECHNOLOGY

\title{
Evaluation of an Evasive Manoeuvre Assistance System at Imminent Side Collisions
}

Downloaded from: https://research.chalmers.se, 2023-04-26 09:55 UTC

Citation for the original published paper (version of record):

Yang, D., Jonasson, M., Halleröd, T. et al (2016). Evaluation of an Evasive Manoeuvre Assistance

System at Imminent Side Collisions. AVEC 16

N.B. When citing this work, cite the original published paper. 


\title{
Evaluation of an Evasive Manoeuvre Assistance System at Imminent Side Collisions
}

\author{
D. Yang \\ Volvo Cars, Active Safety and Vehicle Dynamics Functions, Gothenburg, SE-405 31, Sweden. \\ M. Jonasson \\ Volvo Cars, Active Safety and Vehicle Dynamics Functions, Gothenburg, SE-405 31, Sweden. \\ The Royal Institute of Technology (KTH), Vehicle Dynamics, \\ Department of Aeronautical and Vehicle Engineering, Stockholm, SE-10044, Sweden. \\ T. Halleröd \\ Volvo Cars, Active Safety and Vehicle Dynamics Functions, Gothenburg, SE-405 31, Sweden. \\ R. Johansson \\ Volvo Cars, Safety Centre, Gothenburg, SE-405 31, Sweden.
}

\begin{abstract}
In this paper, the performance of an Evasive Manoeuvre Assistance System is evaluated on the test track, where an imminent half-overlapping side collision scenario is reconstructed. The control function here aims to reduce the steering effort for an emergency swerve in front of obstacle and to ease the following recovery into the driver perceived safe zone. This is realized by combined differential braking and steering torque overlay, which improves the agreement between steering input and vehicle response. Preliminary test results have shown that the function has a great potential to reduce collision risk at the presence of suddenly appeared obstacle in front.
\end{abstract}

\section{INTRODUCTION}

Avoidance of obstacles that suddenly appears outside driver's comfort zone can be handled by reducing vehicle longitudinal speed, or to perform a yaw or sideward motion. Too quick appearance of the obstacle and limited deceleration efficiency without driver initiating and increasing braking may make it impossible to avoid the imminent collision by only reducing the vehicle speed. On the other hand, swerving around the obstacle by combined braking and steering can be more effective to avoid such collision. This type of swerving motion in crash-imminent situations is usually called an evasive manoeuvre.

However, this emergency evasive manoeuvre requires the driver to be very alert and skillful to execute brake and steer action rapidly, appropriately and simultaneously. It is more than common that normal human drivers tend to apply too little or too much steer in facing the forthcoming hazards, with noticeable delays to initiate braking as well (Malaterre, Ferrandez, Fleury, \& Lechner 1988, McGehee, Mazzae, \& Baldwin 1999). The conventional ESC system is designed to assist the driver to maintain vehicle stability assuming correct steering angle from driver. With insufficiently good steering input, ESC would not be the best choice to assist driver to perform a successfully evasive manoeuvre. In addition, conventional ESC does not use environmental sensors, such as camera, to detect threats.

Thereby, the control strategy, Evasive Manoeuvre Assistance System (EMAS) was introduced that aims to make the manoeuvre safer and more effective (Gurov, Sengupta, Jonasson, \& Drugge 2014). The Fig. 1 below illustrates the working principle of EMAS in a rear-end collision avoidance scenario. In this paper, a generic EMAS functionality is developed in a fast-prototyping test vehicle and this implementation is achieved in order to study the potential benefit and risk of EMAS in real-world accident situation. EMAS here will not control the vehicle autonomously. The control must be initiated by the driver, who should be in the loop and be able to override EMAS all the time. This implies that driver takes the responsibility in the control of the vehicle motion.

Previous accident statistics have shown that most drivers do not steer in rear-end collisions although about $50 \%$ the collisions can be avoided by turning around object (Eckert, Hartmann, Sevenich, \& Rieth 2011, Fausten 2010). Simulator studies have suggested that more than $50 \%$ of people chose to steer at imminent side collisions, e.g. with entering ve- 


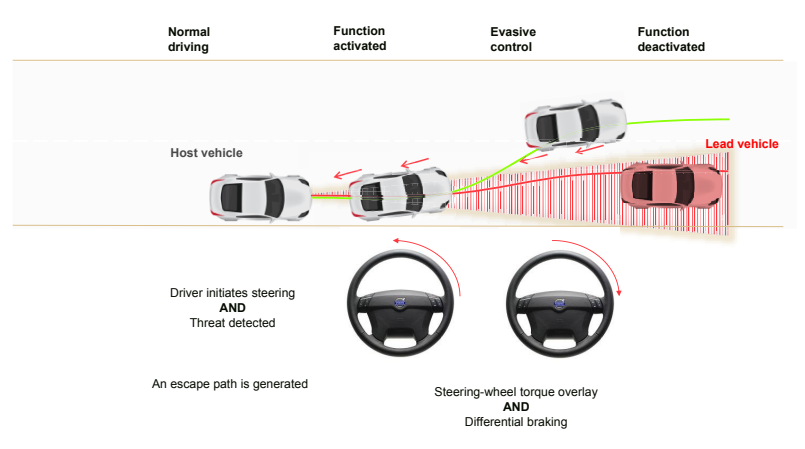

Figure 1: Evasive Manoeuvre Assistance System.

hicle at intersections (Schittenhelm 2005). Reports of under-utilization of vehicle capabilities at crashimminent manoeuvre were reviewed; drivers are more likely to brake than to steer, while the optimal maneuver is more frequently steering alone, or steering combined with braking, rather than braking alone (Adams 1994). In the present paper, an imminent side-collision scenario is reconstructed on the test track that most drivers would choose to steer. Fig.2 photographs the test scenario, where a large-sized box is triggered and tipped over into the driving lane when the vehicle passes a certain reference point. The EMAS function was thereby evaluated under realworld collision avoidance circumstances.

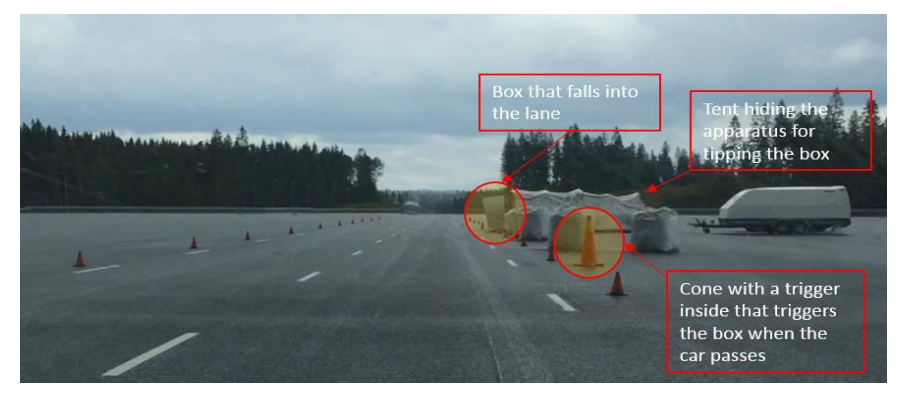

Figure 2: Photograph of test scenario with a suddenly tippedover obstacle on a two-lane road.

\section{FUNCTION DESIGN}

The EMAS function is highly integrated with a class of Volvo's vehicle stability control functions on board, e.g. Active Yaw Control (AYC), Advanced Stability Control (ASC), Roll Stability Control (RSC) etc. At this stage, EMAS also shares certain control components such as friction estimator, vehicle states estimator, vehicle and tyre parameter estimator, vehicle model etc. with aforementioned functions. The coupling with other collision avoidance functions such as Autonomous Emergency Braking (AEB) is also within the scope of the function design.

The activation condition of EMAS function is an ongoing driver initiated side-motion, combined with the presence of a forthcoming collision and a feasible escape path. EMAS will thereby make use of environmental sensors like radars and cameras. The priori information that a single lane change is mostly likely to occur is given by:

- assessed threat in front and

- feasible escape path and

- driver initiated side-motion by steering

The control action exists as a feed-forward controller which requests brake pressures on the inner wheels and torque overlay on the steering wheel. The control inputs are proportional to the desired yaw moment calculated from the reference front axle side slip angle. In this case the controller strives to follow a driver preferred yaw motion. That is to say, EMAS predicts which heading direction the driver would like to control the vehicle towards. In the experiment, the steering wheel torque was applied by the standard Electric Power Assisted Steering (EPAS) system that allows maximum $2.5 \mathrm{Nm}$ torque. The maximum brake pressure was limited by the desired wheel slips specified by the controller, together with the standard wheel slip regulator, e.g. Anti-lock Brake System (ABS). Thereafter, the function is promptly deactivated if:

- the threat becomes invalidated or

- the reference path is found to be infeasible due to e.g. limited space, too low road friction $o r$

- the driver stopped side-motion by steering or

- the underlying vehicle instability is detected.

\section{HYPOTHESIS}

Before the experiment, a number of hypothesis were drawn from the experience of closed-loop simulations with a driver and vehicle model. Fig.3 below shows an example of the simulated path swerving around the obstacle with/without EMAS ( $v_{x}=80 \mathrm{~km} / \mathrm{h}$ ). The driver model was designed for a typical evasive manoeuvre; it was calibrated with vehicle test data and represents a skillful and attentive driver (Wang \& Lokur 2016). It is, for instance, noticed that the driver gained more lateral displacement with the function active. The three hypothesis are:

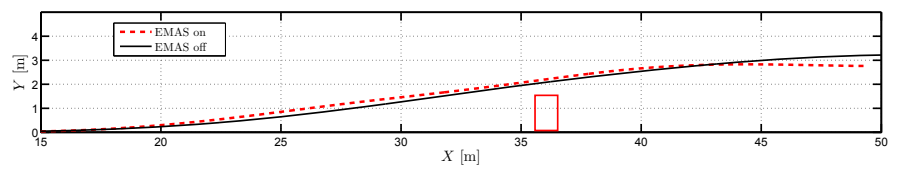

Figure 3: An example path with/without EMAS using a calibrated driver model in simulation. Red box: suddenly appearing obstacle.

- Hypothesis 1: System increases the probability for the driver to avoid the collision. This implies that the function helps the driver move the vehicle more laterally in front of the obstacle. 
- Hypothesis 2: System does not increase risk for the driver to lose control on the vehicle motion. This implies that the driver easily controls the vehicle in the desired lane after avoiding the obstacle.

- Hypothesis 3: System will not intervene the driver too intrusively. This implies that the driver will not feel any disturbance from the function intervention. The driver, vehicle and EMAS controller performs seamless interaction at the emergency situation.

\section{METHOD}

\subsection{Experimental set-up}

The experiment was carried out at the AstaZero (Active Safety Test Area and Zero) proving ground near Gothenburg in Sweden. The Multi Lane (ML) and High Speed Area (HSA) were closed exclusively for the experiment, see the blue circled area in Figure 4.

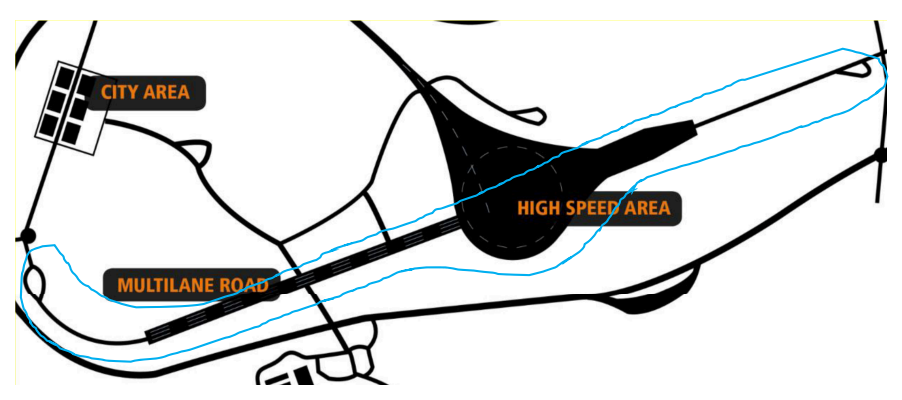

Figure 4: Overview of the used test track (blue circled area). Scale 1:8000.

In total, 27 test subjects were recruited within the Volvo Cars Group, where 12 were women and 15 were men. The age is distributed such that approx. $20 \%$ were younger than 30 years old, $20 \%$ were older than 50 years old, while the rest are between 30 and 50 years old. All drivers have hold their Swedish driver license for at least 5 years with no less than $5000 \mathrm{~km}$ miles driven during the past year. No professional test drivers were involved. We have also excluded subjects who have had whiplash or neck or heart problems. Those who have participated in the development or tests of any collision avoidance systems e.g. EMAS, Lane Keeping Aid etc. were also filtered out.

The control algorithm was implemented in a Volvo S60 sedan test vehicle, which has Cruise Control system that can keep the entering speed identical between subjects. The vehicle was equipped with DEWESoft ${ }^{\circledR}$ measurement package, including IMU (Inertia Measurement Unit) and cameras. In DEWESoft ${ }^{\circledR}$, a virtual lane was laid out in the middle of the actual driving lane on the test track. This could provide a measurement signal containing the heading angle relative to the lane. A virtual spot was also created at the position where the box fell down on the HSA; this gave two signals of the $X$ and $Y$ distance between the test vehicle and the fallen box.

\subsection{Test Scenario}

The real-world scenario reconstructed here in the experiment is a typical pull-out event at the intersections or T-junctions of country roads. The challenge during our test scenario development was: due to safety reasons, enough open driving space should be allowed for this type of crash-imminent evasive manoeuvre; on the other hand, for best fidelity, road constraints should be laid out in a way that driver feels like driving on a real road. Since the scenario also requires a surprising pull-out obstacle, all equipments that trigger the obstacle need to be disguised and explained in a natural way.

The test scenario is illustrated in the Fig.5 below. It is set up at the HSA track which has plenty of space in case of emergency. The straight lane marking was extended from the ML track to HSA so that a two-lane road was reconstructed there. The obstacle was designed as a plastic cubic skeleton box with white plastic cover on one side. A light trigger was positioned inside a cone which is about $22 \mathrm{~m}$ away from the up-coming obstacle; this gives approx. $1 \mathrm{~s}$ time-to-collision (TTC) as the obstacle will be tipped over onto the driving lane once the vehicle passes the trigger. All subjects drove with the Cruise Control function active at $80 \mathrm{~km} / \mathrm{h}$. The single lane width was $3.25 \mathrm{~m}$, and the fallen obstacle makes the free space reduced to $1.8 \mathrm{~m}$. Both sides of the road were painted with lane markings and constrained with tight-positioned cones. The scenario appears natural like a normal road repairs site and no test subjects suspected or questioned about this construction.

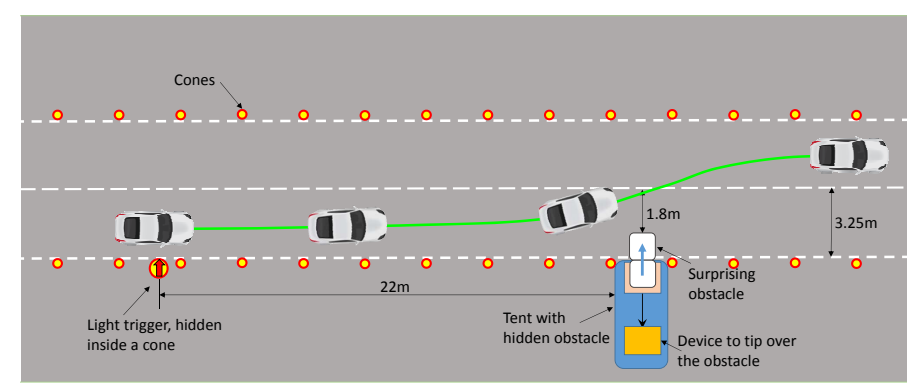

Figure 5: Test scenario of an imminent half-overlapping side collision with a suddenly tipped-over obstacle on a two-lane road.

It is worth mentioning that a pilot testing were carried out before the final experiment, so as to determine the best parameters e.g. entering speed, TTC, overlapping distance etc. The criteria of being best is to make it necessary to steer in order to avoid the collision, and 
the steering activity should be in a sufficiently evasive manner that can trigger the EMAS function. Previous study has shown that upon imminent side collisions, drivers intend to steer around obstacles given a shorter TTC and less overlapping (Eckert, Hartmann, Sevenich, \& Rieth 2011). The size of the obstacle was therefore determined such that it merely creates a half-overlapping scenario. The TTC was thereafter adapted to $1 \mathrm{~s}$ so that drivers would need to steer instead of braking alone. Furthermore, the fallen obstacle scenario here was considered superior to the common dummy-on-trail scenario that it could generate sufficient as well as identical overlapping over the test runs.

\subsection{Test procedures}

The experiment has a between-group design, which has 13 test subjects for each group with EMAS on and off. The driving throughout the entire experiment was accompanied by a test leader on the passenger seat. Before executing the scenario elaborated above, the driver was offered to take a number of training runs within the blue circled area in Fig.4. This is to ensure that driver gets relatively familiar with the test vehicle, as well as the road conditions etc. In the mean time, the spacious HSA track enables the driver to test various braking and steering manoeuvres there.

After getting into the car, the driver got a short introduction from the test leader about the secondary task that he/she was supposed to perform during the drive. The secondary task was to read numbers from a small screen placed above the passenger seat. It was five numbers (1-9) that lit up one by one with $0.5 \mathrm{~s}$ in between. The secondary task started by a recorded voice saying "Read the numbers now". The driver was then told that the purpose of the study was to investigate gaze and steering behaviours during secondary tasks. They were not told anything about the collision avoidance event. The secondary task was also designed in a way that the driver would feel naturalistic like in daily driving and be distracted to certain extent before the intrusion of surprising obstacle. At the end of the experiment, the driver filled out a questionnaire about the fidelity of the reconstructed driving session, evaluation of own driving experience and performance, as well as the interaction with the EMAS function (if active).

\section{FUNCTION EVALUATION}

The function performance is evaluated here according to the three hypothesis anticipated in Section 3.

\subsection{Collision risk}

Here two measures are used to indicate the risk of collision with/without the EMAS function. One is the vehicle lateral displacement $\left(Y_{\text {box }}\right)$ measured when the
Table 1: Comparison of collision risk with/without EMAS function. $Y_{\text {box }}$ : vehicle lateral displacement when passing obstacle; $\psi_{\max }$ : max yaw angle until passing obstacle.

\begin{tabular}{llllll}
\hline & \multicolumn{2}{c}{ mean } & & \multicolumn{2}{c}{ standard deviation } \\
\cline { 2 - 3 } \cline { 5 - 6 } Measure & with & without & & with & without \\
\hline$Y_{\text {box }}[\mathrm{m}]$ & 0.56 & 0.22 & & 0.39 & 0.22 \\
$\psi_{\max }[\mathrm{deg}]$ & 5.1 & 3.1 & & 2.2 & 2.6 \\
\hline
\end{tabular}

vehicle front-right corner passing the obstacle rearleft corner, along the direction which is perpendicular to the current vehicle yaw angle. While the other one is to measure the maximum yaw angle $\left(\psi_{\max }\right)$ the vehicle reached until passing the obstacle, which indicates the enhancement of yaw capability with EMAS active. Table 1 has summarized the mean and standard deviation values of the these two measures. It can be seen that with EMAS, the $Y_{\text {box }}$ was almost tripled with increased $\psi_{\max }$ even if the absolute value was not particularly large in this case. It is noticed that the standard deviations among the test subjects were relatively high, so it indicates a need of more subjects in order to reach higher statistic confidence.

Fig. 6 below shows the mean value of trajectories at the vehicle front-right corner. In total, five drivers without EMAS had collision with the obstacle, and one driver could avoid the collision by staying extreme near the left lane marking before steering around the obstacle; while one driver with EMAS had a slight side swipe with the obstacle. This result has clearly indicated the effectiveness of EMAS with respect to reduced collision risk. Considering the more severe consequence of small-overlapping accidents as compared to the full-overlapping ones, the benefit of reducing the collision risk here can not be underestimated. It is also interesting to find that after avoiding the obstacle, both groups were able to control the vehicle towards the same perceived safe zone, e.g. about $1 \mathrm{~m}$ beside the original lane. See Section 5.2 below with closer analysis of the vehicle stability throughout the entire event.

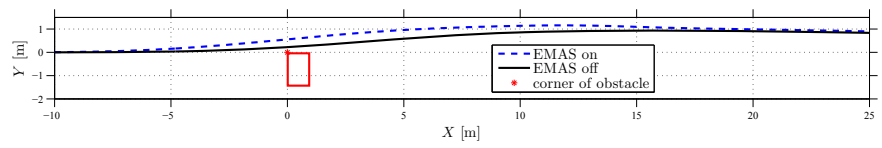

Figure 6: Trajectory (mean value) of all test subjects at the vehicle front-right corner, with/without EMAS function. Red box: suddenly appearing obstacle.

\subsection{Vehicle stability}

The EMAS function is designed to assist the driver to reduce the risk of imminent collisions, without introducing vehicle instability in any sense. Hence the maximum side slip angles at both front and rear axle, yaw rate and lateral acceleration were observed. Table 2 has summarized the mean and standard deviations of these four measures. It shows that under this scenario, EMAS could safely increase the yaw rate 
Table 2: Comparison of vehicle stability measures with/without EMAS function. $\beta_{\text {fmax }}$ : $\max$ front side slip angle; $\beta_{\text {rmax }}$ : max rear side slip angle; $\psi_{\max }$ : max yaw rate; $a_{y \max }: \max$ lateral acceleration.

\begin{tabular}{llllll}
\hline & \multicolumn{2}{c}{ mean } & & \multicolumn{2}{c}{ standard deviation } \\
\cline { 2 - 3 } \cline { 5 - 6 } Measure & with & without & & with & without \\
\hline$\beta_{\text {fmax }}[\mathrm{deg}]$ & 1.44 & 0.79 & & 0.43 & 0.54 \\
$\beta_{\text {rmax }}[\mathrm{deg}]$ & 1.99 & 0.82 & & 0.74 & 0.63 \\
$\dot{\psi}_{\max }[\mathrm{deg} / \mathrm{s}]$ & 18.14 & 9.61 & & 4.19 & 6.60 \\
$a_{y \max }\left[\mathrm{m} / \mathrm{s}^{2}\right]$ & 6.02 & 3.35 & & 1.22 & 1.95 \\
\hline
\end{tabular}

and lateral acceleration while still keeping the tyre side slip angles within the linear region.

\subsection{Driver response and experience}

Under the circumstances of such emergent and surprising crash-imminent event, we aim to apply the assistance timely and appropriately without disturbing the driver's normal driving skill. The driver should feel intuitively with the function intervention. Therefore, both objective and subjective measures are used here to evaluate the function-driver interaction.

We define the state start of steering as the steering wheel angle becomes over $15 \mathrm{deg}$. It is found that with EMAS driver tends to reach the start of steering earlier, i.e. at $0.37 \mathrm{~s}$ compared to $0.57 \mathrm{~s}$ without EMAS. Fig. 7 below displays the objective measures of steering activity, with respect to torsion bar torque, steering wheel angle and angular velocity. The mean value of max torsion bar torque is shown to be lower with EMAS than without, which indicates the reduced steering effort from the driver. On the other hand, all of the three measures have a phase lead when EMAS was on; this again gives the function benefit in term of time winning and thus more agile during the emergent lateral motion.

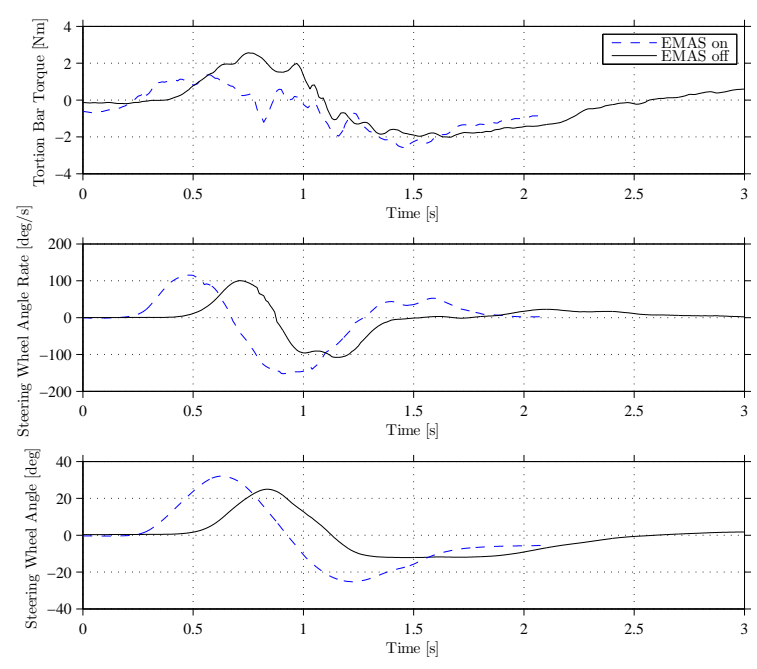

Figure 7: Steering activity (mean value) of all test subjects with/without EMAS function.

Subjective evaluation was drawn from the answers in questionnaires. Generally, drivers have experienced no differences in terms of steering feeling, controllability of the vehicle and driving performance. Nevertheless, two drivers without EMAS expressed their suspicion afterwards that certain ABS and steering assistance system was active; while six drivers with EMAS has felt about steering and braking interventions from some type of stability control systems. The drivers did not note any unpleasantness with EMAS active during the manoeuvre.

\section{SELECTED EXAMPLES}

In this section, two representative examples of the test subjects with/without EMAS function are presented. The function was triggered at $t=0.5 \mathrm{~s}$. Fig. 8 shows the full trajectory where a much stronger sideswerving motion is exhibited with EMAS. The driver without EMAS has collided with the obstacle while the one with EMAS could narrowly miss the collision. It should be noted that TTC $=1 \mathrm{~s}$ was so short that most drivers were at the risk boundary of collision. Fig.9 shows the typical vehicle states during such evasive manoeuvre, which indicates that the EMAS function could enhance the lateral displacement in a safe and stable way. Fig. 10 shows the steering wheel angle as well as the EPAS torque request by the EMAS function. Fig.11 displays the driver brake activities of these two examples that, the driver with EMAS started to brake down the vehicle by the end of the steering manoeuvre, while it took much more time for the driver without EMAS to start braking. This in some sense implies that EMAS could release the driver from the complex evasive manoeuvre earlier and thus ease the following recovery into the safe zone.

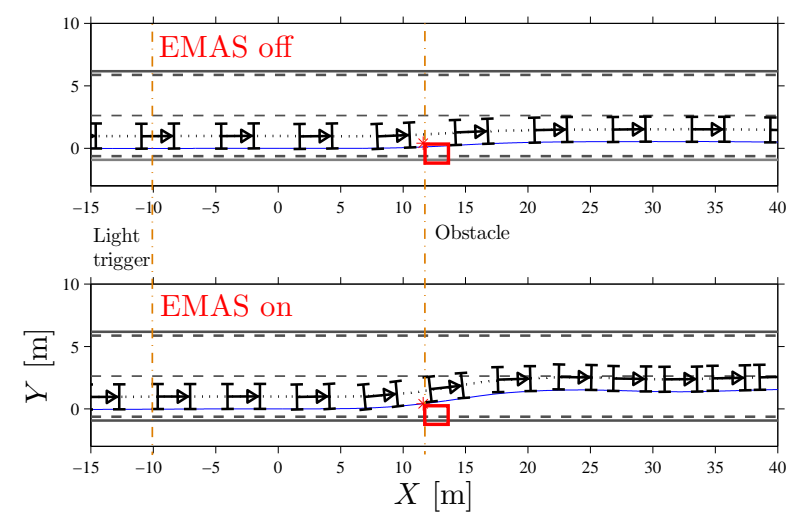

Figure 8: Trajectory of two exemplar test subjects with/without EMAS function. Blue curve: trajectory of the vehicle front-right corner. Red box: suddenly appearing obstacle.

\section{CONCLUSIONS}

In this paper, an evasive manoeuvre assist system (EMAS) was evaluated at a crash-imminent 

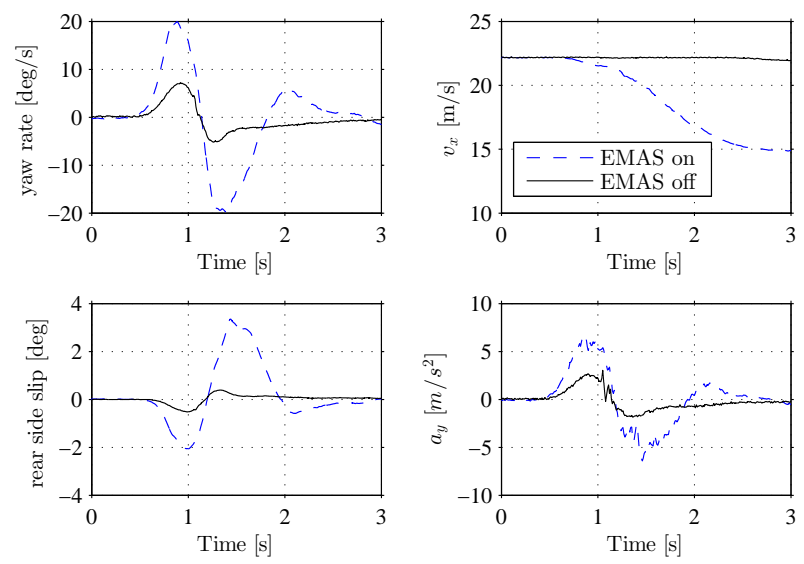

Figure 9: Vehicle states of two exemplar test subjects with/without EMAS function.

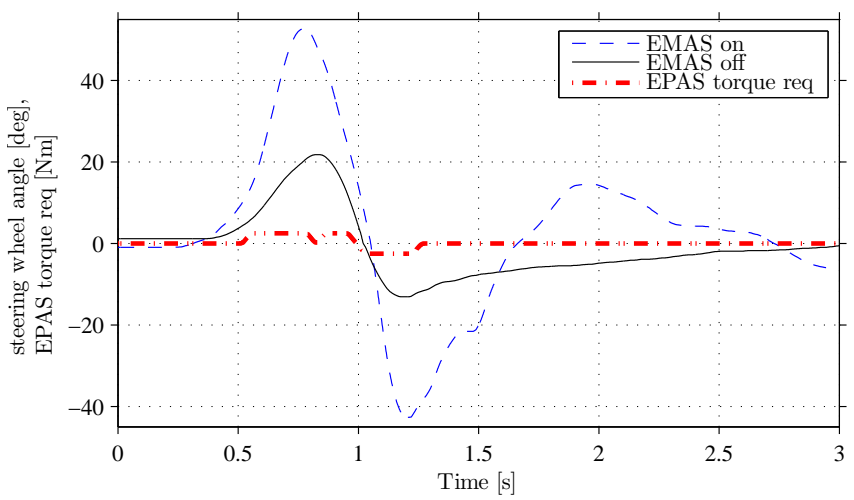

Figure 10: Steering wheel angle and EPAS torque request of two exemplar test subjects with/without EMAS function.

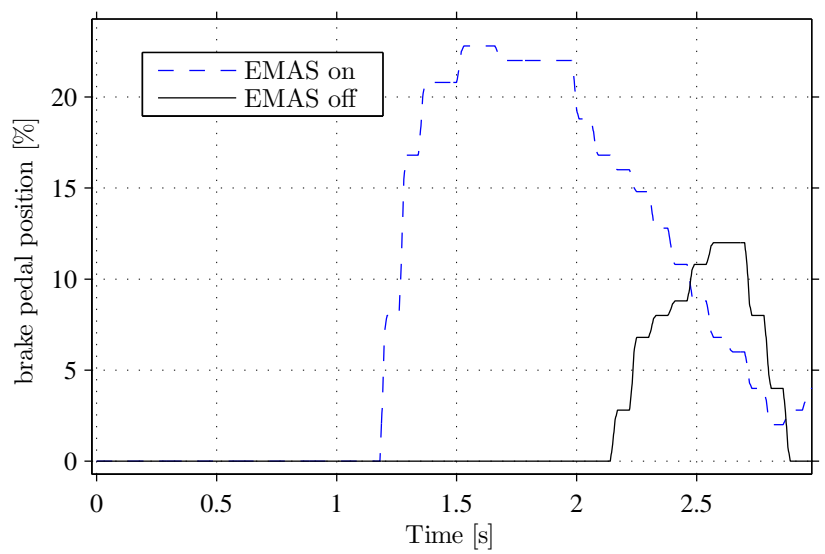

Figure 11: Brake pedal position of two exemplar test subjects with/without EMAS function.

scenario reconstructed at the test track. A halfoverlapping side collision at the intersection of typical European country roads was reconstructed with real-world perspectives. The control function actively applies individual wheel braking and steering wheel torque overlay interventions so as to assist the driver to avoid the collision. Objective and subjective evaluation criteria were built according to three hypothesis drawn from previous results in the closed-loop simulations with a scenario-calibrated driver model.

It is found that the EMAS function could significantly reduce the collision risk under this type of half-overlapping accidents. This benefit is achieved by enhanced yaw and lateral motion capability, without damaging the bounded vehicle stability. EMAS is proven to have reduced the steering effort in swerving around the suddenly appearing obstacle in front. The function could appropriately and effectively assist a variation of drivers with different driving skills. The evaluation method developed here is considered novel that is applicable to a fleet of vehicle collision avoidance systems.

Future work remains to test the function at scenarios including different road users, e.g. oncoming vehicles and pedestrians from side. More test subjects could be involved in a larger scale experiment in order to increase the statistical confidence. The present EMAS function is expected to further adapt to the drivers who steer not too little but on the contrary too much, for instance, on the icy roads.

\section{ACKNOWLEDGMENTS}

This work was in part financed by the Swedish national competence centre ViP (Virtual Processing and Assessment by Simulation) driving simulation centre. The authors would like to extend appreciation to Mats Petersson for the research project management, and Anders Axelson for the expert insights during test method development.

\section{REFERENCES}

Adams, L. D. (1994). Review of the literature on obstacle avoidance maneuvers: braking versus steering. University of Michigan Transportation Research Institute, Ann Arbor, USA. Report No. UMTRI-94-19.

Eckert, A., B. Hartmann, M. Sevenich, \& P. E. Rieth (2011). Emergency steer and brake assist - a systematic approach for system integration of two complementary driver assistance systems. Proc. of the 22nd International Technical Conference on the Enhanced Safety of Vehicles (ESV), Washington $D C, U S A$.

Fausten, M. (2010). Accident avoidance by evasive manoeuvres - challenges and steps towards technical solutions. http://www.ftm.mw.tum.de.

Gurov, A., A. Sengupta, M. Jonasson, \& L. Drugge (2014). Collision avoidance driver assistance system using combined active braking and steering. Proc. of the 12th International Symposium on Advanced Vehicle Control (AVEC14), Tokyo, Japan.

Malaterre, G., F. Ferrandez, D. Fleury, \& D. Lechner (1988). Decision making in emergency situations. Ergonomics 31(4), 643-655.

McGehee, D. V., E. N. Mazzae, \& G. H. S. Baldwin (1999). Examination of drivers' collision avoidance behavior using conventional and antilock brake systems on the iowa driving simulator. Human Factors \& Vehicle Safety Research. NHTSA report No. 6-15-1999.

Schittenhelm, H. (2005). Fahrverhalten und reaktionszeiten in kritischen situationen. VDI-Bericht 1911. Düsseldorf, Germany.

Wang, Y. \& P. Lokur (2016). Effectiveness of evasive manoeuver assist function for different driver skills. Chalmers University of Technology, Gothenborg, Sweden. Master thesis No. EX006/2016. 